\title{
Glaucoma, the disease that never sleeps
}

\author{
Glaucoma a doença que nunca dorme
}

G laucoma is the leading cause of irreversible blindness worldwide and a major public health problem ${ }^{(1,2)}$. Despite important advances in the diagnosis and management of glaucoma during the last decades, the fundamental understanding of intraocular pressure (IOP), its only modifiable risk factor, remains elusive. Whereas patients suffering from other chronic diseases, such as diabetes, have been empowered by the availability of round-the-clock monitoring devices, glaucoma patients have to make do with outdated technology available only in the physician's office.

Intraocular pressure is a highly variable biological parameter. The current practice of static IOP measurements during a clinic visits has been widely shown to not adequately reflect the variability of IOP throughout the 24-h period, as only a minority of IOP peaks occur during usual daytime office hours ${ }^{(3)}$. In fact, IOP is highest during the nocturnal sleep period in a majority of glaucoma patients ${ }^{(4)}$. These values go largely undetected and are not taken into account in patient management decisions. The implications of this omission are currently unknown and unquantifiable but may explain a significant amount of those patients who progress despite having a supposedly controlled IOP(5).

The availability of 24-h IOP monitoring technologies has, therefore, been formulated as a major unmet need in ophthalmology and attempts at addressing it were undertaken as long ago as $1958^{(6)}$. In the last decade, there has been considerable progress with the prototype and commercial introduction of continuous 24-h IOP monitoring devices, facilitated by progress in micro- and nano-electromechanical systems. Two approaches are actively pursued, one via contact lenses, the other via implantable sensors. The latter have the advantage to directly measure IOP over long periods, while the former provide a non-invasive alternative for repeated 24-h periods.

At present, the Triggerfish contact lens sensor (CLS; Sensimed, Switzerland) is the only commercially available IOP monitoring device in some parts of the world (currently not in Brazil). It measures strain of a contact lens gauge at a frequency of $20 \mathrm{~Hz}$. It is important to note that the CLS does not measure IOP directly but rather a composite of intraocular pressure, volume and ocular biomechanical properties ${ }^{(7,8,9)}$. While tonometry measures IOP, CLS is thought to be more influenced by intraocular volume with the relationship between the two being curvilinear ${ }^{(10)}$. The interaction of corneal and scleral biomechanical properties and CLS has not been sufficiently studied ${ }^{(11,12)}$. Furthermore, the CLS provides its measurements in $\mathrm{mV}$ equivalents instead of the habitual $\mathrm{mmHg}$-unit. All the above factors have prevented direct comparisons between CLS and tonometry and have rendered clinical interpretation of its readings challenging. The major value of the device is that it can record the IOP related profile in a real-life setting for up to 24 hours including during undisturbed sleep ${ }^{(13)}$. Documenting relative changes of IOP related events and their timing may yet prove to be of similar importance than absolute IOP levels. In addition to the CLS, other non-invasive devices are currently being developed ${ }^{(14,15)}$.

In 2011, an implantable telemetric pressure transducer system to continuously monitor IOP was tested in non-human primates and shown to provide accurate measurements ${ }^{(16)}$. For the moment, however, the extensive surgery required for its implantation precludes use in humans. Combining IOP sensors with IOLs used in routine cataract surgery may facilitate patient acceptance. More recently, a foldable wireless IOP transducer using pressure sensor cells to measure IOP (Implandata, Germany) has been tested in rabbits and humans ${ }^{(17,18)}$. The device is designed to be implanted in the ciliary sulcus. Currently, it is being tested in clinical trials. Preliminary results after one year demonstrate the device to be generally safe and capable to obtain reliable measurements in a majority of eyes ${ }^{(19)}$. The main adverse events observed are iris chafing and pigment liberation. Limitations of intraocular sensors are related to safety issues associated with surgical implantation and their long term tolerance. The potential for device failure after implantation, leakage of potentially toxic materials as well as inaccuracy of measurements due to signal drift over time with the need of subsequent intervention for re-calibration constitute some other challenges. Before these technologies can obtain regulatory approval and widespread acceptance for use in humans, these risks have to be addressed.

The field of 24-h IOP monitoring has made great strides in recent years and it is expected that other devices will become available in the near future. At present, however, it is unclear whether the contact lens approach or the intraocular sensors will become the new IOP "gold standard". Both may co-exist as complimentary to each other. In the meantime, the glaucomatologists wish-list for the perfect 24-h IOP monitoring device would include the following: 1) well-tolerated and safe; 2$)$ accurate; 3 ) high-frequency of measurements $(>40 \mathrm{~Hz})$ to detect ultra short events; 4$)$ affordable and 5) capable to store and release hypotensive drugs as needed. 
Once such devices become available for routine clinical use, it will become obvious that we lack true understanding of the relationship between IOP and glaucoma:

- Does nocturnal IOP have the same impact on glaucoma as diurnal IOP?

Which IOP parameters are most relevant to glaucoma: mean, peak, short or long-term variability?

Are there specific circadian IOP patterns that are related to glaucoma development and may predict its future progression?

It is likely that the use of 24-h IOP monitoring will improve understanding and management of glaucoma. Ultimately, clinically-driven 24-h IOP data may lead to chrono-therapeutic delivery of glaucoma medications instead of the current practice of standard scheduling of drop instillation. In the meantime, clinicians are encouraged to obtain as many daily (and nightly) IOP measurements, possibly augmented with provocative testing, such as water loading, as possible to gain a better understanding of a patient's IOP dynamics and to personalize his treatment.

Kawed Mansouri, M.D., M.P.H.

Glaucoma Center, Montchoisi Clinic, Genolier Swiss Vision Network, Lausanne, Switzerland Department of Ophthalmology, University of Colorado School of Medicine, Denver, USA

Conflict of interest: Research and financial support from Sensimed AG

\section{REFERENCES}

1. Resnikoff S, Pascolini D, Etya'ale D, Kocur I, Pararajasegaram R, Pokharel GP, et al. Global data on visual impairment in the year 2002. Bull World Health Organ. 2004;82(11):844-51.

2. Mansouri K, Medeiros FA, Weinreb RN. Global rates of glaucoma surgery. Graefe's archive for clinical and experimental ophthalmology = Albrecht von Graefes Archiv fur klinische und experimentelle Ophthalmologie. 2013;251(11):2609-15.

3. Nakakura S, Nomura Y, Ataka S, Shiraki K. Relation between office intraocular pressure and 24-hour intraocular pressure in patients with primary open-angle glaucoma treated with a combination of topical antiglaucoma eye drops. Journal of glaucoma. 2007;16(2):201-4.

4. Liu JH, Zhang X, Kripke DF, Weinreb RN. Twenty-four-hour intraocular pressure pattern associated with early glaucomatous changes. Investigative ophthalmology \& visual science. 2003;44(4):1586-90.

5. Mansouri K, Weinreb RN. Meeting an unmet need in glaucoma: continuous 24-h monitoring of intraocular pressure. Expert review of medical devices. 2012;9(3):225-31.

6. Maurice DM. A recording tonometer. The British journal of ophthalmology. 1958;42(6):321-35.

7. Hjortdal JO, Jensen PK. In vitro measurement of corneal strain, thickness, and curvature using digital image processing. Acta ophthalmologica Scandinavica. 1995;73(1):5-11

8. Leonardi M, Leuenberger P, Bertrand D, Bertsch A, Renaud P. First steps toward noninvasive intraocular pressure monitoring with a sensing contact lens. Investigative ophthalmology \& visual science. 2004;45(9):3113-7.

9. Mansouri K, Weinreb R. Continuous 24-hour intraocular pressure monitoring for glaucoma-time for a paradigm change. Swiss Med Wkly. 2012;142:w13545.

10. Silver DM, Geyer O. Pressure-volume relation for the living human eye. Current eye research. 2000;20(2):115-20.

11. Hubanova R, Aptel F, Chiquet C, Mottet B, Romanet JP. Effect of overnight wear of the Triggerfish((R)) sensor on corneal thickness measured by Visante $((\mathrm{R}))$ anterior segment optical coherence tomography. Acta Ophthalmol. 2014;92(2):e119-23.

12. Freiberg FJ, Lindell J, Thederan LA, Leippi S, Shen Y, Klink T. Corneal thickness after overnight wear of an intraocular pressure fluctuation contact lens sensor. Acta Ophthalmol. 2012;90(7):e534-9.

13. Mansouri K, Shaarawy T. Continuous intraocular pressure monitoring with a wireless ocular telemetry sensor: initial clinical experience in patients with open angle glaucoma. The British journal of ophthalmology. 2011;95(5):627-9.

14. Margalit I, Beiderman Y, Skaat A, Rosenfeld E, Belkin M, Tornow RP, et al. New method for remote and repeatable monitoring of intraocular pressure variations. Journal of biomedical optics. 2014;19(2):027002.

15. Chen GZ, Chan IS, Leung LK, Lam DC. Soft wearable contact lens sensor for continuous intraocular pressure monitoring. Medical engineering \& physics. 2014;36(9):1134-9.

16. Downs JC, Burgoyne CF, Seigfreid WP, Reynaud JF, Strouthidis NG, Sallee V. 24-hour IOP telemetry in the nonhuman primate: implant system performance and initial characterization of IOP at multiple timescales. Investigative ophthalmology \& visual science. 2011;52(10):7365-75

17. Melki S, Todani A, Cherfan G. An implantable intraocular pressure transducer: initial safety outcomes. JAMA ophthalmology. 2014;132(10):1221-5.

18. Todani A, Behlau I, Fava MA, Cade F, Cherfan DG, Zakka FR, et al. Intraocular pressure measurement by radio wave telemetry. Investigative ophthalmology \& visual science. 2011;52(13):9573-80.

19. Koutsonas A, Walter P, Roessler G, Plange N. Implantation of a Novel Telemetric Intraocular Pressure Sensor in Patients With Glaucoma (ARGOS Study): 1-Year Results. Investigative ophthalmology \& visual science. 2015;56(2):1063-9. 